\title{
From Diffusions on Graphs to Markov Chains via Asymptotic State Lumping
}

\author{
Adam Bobrowski
}

\begin{abstract}
We show that fast diffusions on finite graphs with semi permeable membranes on vertices may be approximated by finite-state Markov chains provided the related permeability coefficients are appropriately small. The convergence theorem involves a singular perturbation with singularity in both operator and boundary/transmission conditions, and the related semigroups of operators converge in an irregular manner. The result is motivated by recent models of synaptic depression.
\end{abstract}

\section{Intuition}

Imagine a finite graph $\mathcal{G}$ without loops and a Markov process on $\mathcal{G}$ obeying the following informal rules.

- While on the $i$ th edge, imagined as a $C^{1}$ curve in $\mathbb{R}^{3}$, the process behaves like a one-dimensional Brownian motion with variance $\sigma_{i}>0$.

- Graph's vertices are semipermeable membranes, allowing communication between the edges; permeability coefficients $p_{i j}$, describing the possibility to filter through the membrane from the $i$ th to the $j$ th edge, depend on the edges. In particular, $p_{i j}$ is in general different from $p_{j i}$. At each vertex, the process may also be killed and removed from the state space.

Now, suppose the diffusion's speed increases while membranes' permeability decreases (i.e., $\sigma_{i} \rightarrow \infty$ and $p_{i j} \rightarrow 0$ ). As a result, points in each edge communicate almost immediately and in the limit are lumped together, but the membranes prevent lumping of points from different edges. We will show, nevertheless, that the assumption that the rate with which permeability coefficients tend to zero is the same as the rate with which the diffusion coefficients tend to infinity, leads to a limit process in which communication between lumped edges is possible. The lumped edges form then the vertices in the

This research was supported by the Polish Government, grant number 6081/B/H03/2011/40. 

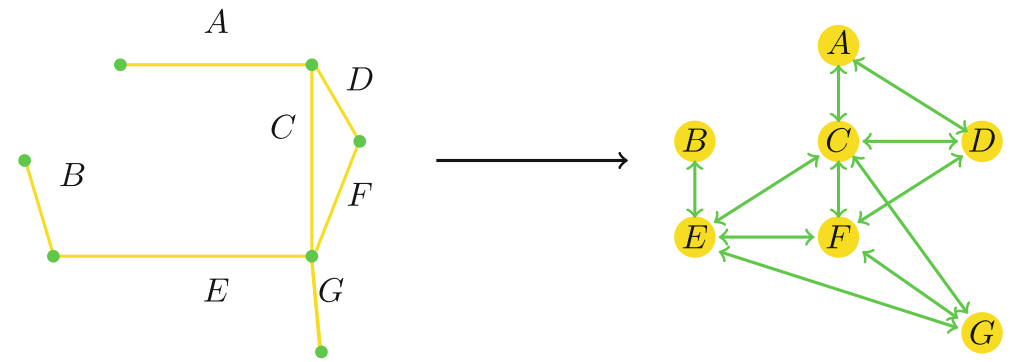

Figure 1. From diffusion on $\mathcal{G}$ to a Markov chain on the vertices of the line graph of $\mathcal{G}$; edges 'shrink' to vertices, vertices 'split' into edges

so-called line graph of $\mathcal{G}$ (see [10]) and communicate as the states of a Markov chain with jumps' intensities directly proportional to permeability coefficients $p_{i j}$ and the diffusion coefficients $\sigma_{i}$ and inversely proportional to the edges' lengths (see Fig. 1 and Theorem 2.2). The assumption on the rate is important: if diffusion coefficients tend to infinity slower than the permeability coefficients tend to zero, there is no communication between the vertices in the limit line graph, and in the opposite case, all points of the original graph are lumped together, and nothing interesting happens.

This procedure may also be reversed: given a finite-state Markov chain, we may find a graph $\mathcal{G}$ and construct a fast diffusion on $\mathcal{G}$ approximating the chain.

Our result is somewhat similar to that of Wentzel and Freidlin [21,23]. Here, however, motivations are of biological rather than of physical nature. In fact, our main theorem is a natural generalization of the main result of [9], where a connection was established between recent PDE and ODE models of synaptic depression. Compare also the model of kinase activity presented in [26] where, much as in our case, fast diffusion combined with feedback from the boundary results in a singular perturbation and a surprising limit. Examples of interesting biological membranes may also be derived from modeling efflux proteins.

\section{The main theorem}

Let $\mathcal{G}=(\mathcal{V}, \mathcal{E})$ be a finite geometric graph [31] without loops, where $\mathcal{V} \subset \mathbb{R}^{3}$ is the set of vertices, and $\mathcal{E}$ is the set of edges of finite length. The number of edges is $N$, and the edges are seen as $C^{1}$ curves connecting vertices. For $i \in \mathcal{N}:=\{1, \ldots, N\}, L_{i}, R_{i} \in \mathcal{V}$ denote the left and right endpoints of the $i$ th edge $E_{i}$, respectively; the decision which is the left point and which is the right point is made arbitrarily.

Since each vertex $V$ is a semipermeable membrane, the future behavior of the related diffusion process starting at $V$ depends on which side of the membrane it lies. Hence, if $k \leq N$ edges are incident in $V, V$ splits naturally 
into $k$ points. By $V_{i} \in \mathcal{N}$, we denote $V$ as an endpoint of the $i$ th edge (if $V$ is not an endpoint of this edge, we leave $V_{i}$ undefined).

The state space of the diffusion process is the union $S=\bigcup_{i \in \mathcal{N}} E_{i}$ of disjoint edges; as above, even though endpoints of many edges coincide, we treat them as distinct. $S$ is a (disconnected) compact topological space, and the related space of continuous complex functions $C(S)$ is isometrically isomorphic to the Cartesian product $C\left(E_{1}\right) \times \cdots \times C\left(E_{N}\right)$ of spaces of continuous functions on the edges (equipped with the norm $\left.\left\|\left(f_{i}\right)_{i \in \mathcal{N}}\right\|=\max _{i \in \mathcal{N}}\left\|f_{i}\right\|_{C\left(E_{i}\right)}\right)$. On the other hand, each $C\left(E_{i}\right)$ is isometrically isomorphic to the space $C\left[0, d_{i}\right]$ of continuous functions on the interval $\left[0, d_{i}\right]$, where $d_{i}$ is the length of the $i$ th edge: a function $f \in C\left(E_{i}\right)$ is then identified with its image If $\in C\left[0, d_{i}\right]$, given by $I f(t)=f(p)$, where $p$ is the unique point on $E_{i}$, whose distance from the left endpoint of $E_{i}$ (along the edge) is $t \in\left[0, d_{i}\right]$. In particular, we may meaningfully speak of continuously differentiable functions on $E_{i}$; these are simply the images of continuously differentiable functions on $\left[0, d_{i}\right]$.

Let $\sigma \in C(S)$ be defined by $\sigma(p)=\sigma_{i}$, for $p \in E_{i}$, where $\sigma_{i}$ are given positive numbers. We define the operator $A$ in $C(S)$ by

$$
A f=\sigma f^{\prime \prime}
$$

for twice continuously differentiable functions on $C(S)$, satisfying the transmission conditions described below.

For each $i$, let $l_{i}$ and $r_{i}$ be nonnegative numbers describing the possibility of passing through the membrane from the $i$ th edge to the edges incident in the left and right endpoints, respectively. Also, let $l_{i j}$ and $r_{i j}, j \neq i$ be nonnegative numbers satisfying $\sum_{i \neq j} l_{i j} \leq l_{i}$ and $\sum_{i \neq j} r_{i j} \leq r_{i}$. These numbers determine the probability that after filtering through the membrane from the $i$ th edge, a particle will enter the $j$ th edge (see Fig. 2).

By default, if $E_{j}$ is not incident in $L_{i}$, we put $l_{i j}=0$. In particular, by convention $l_{i j} f\left(V_{j}\right)=0$ for $f \in C(S)$, if $V_{j}$ is not defined; the same remark concerns $r_{i j}$. In these notations, the transmission conditions mentioned above are as follows: if $L_{i}=V$, then

$$
f^{\prime}\left(V_{i}\right)=l_{i} f\left(V_{i}\right)-\sum_{j \neq i} l_{i j} f\left(V_{j}\right)
$$

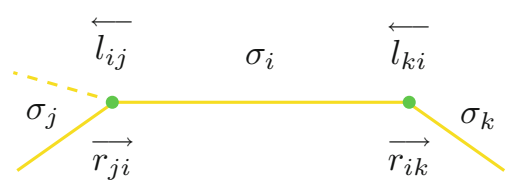

FiguRE 2. $l_{i j} \mathrm{~s}$ and $r_{i j} \mathrm{~s}$ are permeability coefficients of the membranes at vertices and determine probabilities of passing from one edge of the graph to another 
where $f^{\prime}\left(V_{i}\right)$ is the left-hand derivative of $f$ at $V_{i}$, and if $R_{i}=V$, then

$$
-f^{\prime}\left(V_{i}\right)=r_{i} f\left(V_{i}\right)-\sum_{j \neq i} r_{i j} f\left(V_{j}\right),
$$

where $f^{\prime}\left(V_{i}\right)$ is the right-hand derivative of $f$ at $V_{i}$.

These conditions have their origin in the work of Feller [15-18] (see also $[14,29,34])$ and are different in nature from those commonly used in the theory of diffusions on networks $[19,30,31]$, the latter usually involving continuity of $f$ or its derivative (compare, however, Chapter 14 in [31], eq. (14.3)). Their interpretation is that the diffusion process starting at the $i$ th edge 'bounces' from the membrane at the left end, and the time it spends at the membrane is measured by the related Lévy local time $t^{+}$(see $[24,25,33]$ for the definition). Then, at a random time $T$ distributed according to

$$
P(T>t)=\mathrm{e}^{-l_{i} t^{+}}, \quad t \geq 0,
$$

the process filters through the membrane; the probability that it will filter to the $j$ th edge is $\frac{l_{i j}}{l_{i}} ; 1-l_{i}^{-1} \sum_{j \neq i} l_{i j}$ is the probability that the particle is removed from the state space. The behavior at the right end is analogous.

It is appropriate to compare (2.2)-(2.3) with the boundary conditions studied in $[20-23,27,28,30]$ in the context of Feller semigroups, where a characterization of the most general boundary conditions related to a diffusion on a graph is given along with probabilistic description of the process. The main difference between these two approaches is in the state space of the process: in the present paper, because of the membranes at vertices, each vertex splits naturally into several points, representing positions at various sides of the membrane, and the issuing state space $S$ of the process is a disjoint union of the edges. In the papers cited above, the state space is the graph with each vertex being a common point of all the edges incident in this vertex, and the analysis is done in the space $C(\mathcal{G})$ of continuous functions on $\mathcal{G}$. In other words, members of $C(S)$ seen as functions on the graph may have discontinuities of the first type at the edges and in general do not belong to $C(\mathcal{G})$. Probabilistically, a diffusion in the sense considered in the present paper is not a diffusion in the sense of [28], say, as it involves jumps from one copy of a vertex to another copy of the vertex.

In this context, it is perhaps worth stressing that conditions (2.2) and (2.3) are in a sense nonlocal. For example, writing $\delta_{j, V}$ for the Dirac measure concentrated at $V_{j}$, we obtain the following form of $(2.2)$ :

$$
f^{\prime}\left(V_{i}\right)=l_{i} f\left(V_{i}\right)-\int f \mathrm{~d} \mu_{i, L},
$$

where $\mu_{i, L}=\sum_{j \neq i} l_{i j} \delta_{j, V}$. The latter measure is the distribution of the position of a diffusing particle right after it filters from the $i$ th edge through the membrane at its left end $V$, and strictly speaking this position is reached by a jump from $V_{i}$. The nonlocal character of condition (2.4) would become even more clear if $\mu_{i, L}$ were replaced by a measure on the union of edges incident in $V$, and we believe our approximation is robust to such a change. On the 
other hand, intuitionally, (2.4) describes the process of filtering through the membrane at $V$, and $V_{i}$ 's are but copies of the same point, so that no 'real' jumps occur. In particular, replacing $\mu_{i, L}$ by a more general measure would be counterintuitive.

Proposition 2.1. A generates a Feller semigroup in $C(S)$, denoted $\left\{\mathrm{e}^{t A}, t \geq 0\right\}$. The semigroup is conservative iff

$$
\sum_{j \neq i} l_{i j}=l_{i} \text { and } \sum_{j \neq i} r_{i j}=r_{i}, \quad i \in \mathcal{N} .
$$

Theorem 2.2. Let $\left(\kappa_{n}\right)_{n \geq 1}$ be a sequence of positive numbers converging to infinity, and let operators $A_{n}$ be defined by (2.1) with $\sigma$ replaced by $\kappa_{n} \sigma$ and with domain composed of $C^{2}(S)$ functions satisfying the transmission conditions (2.2) and (2.3) with all permeability coefficients (i.e., all $l_{i}, r_{i}, l_{i j}$ and $\left.r_{i j} s\right)$ divided by $\kappa_{n}$. Then,

$$
\lim _{n \rightarrow \infty} \mathrm{e}^{t A_{n}} f=\mathrm{e}^{t Q} P f, \quad f \in C(S), t>0,
$$

where $P$ is the projection of $C(S)$ on the space $C_{0}(S)$ of functions that are constant on each edge, given by $P f=\left(d_{i}^{-1} \int_{E_{i}} f\right)_{i \in \mathcal{N}}$, while $Q$ is the operator in $C_{0}(S)$ which may be identified with the matrix $\left(q_{i j}\right)_{i, j \in \mathcal{N}}$ with $q_{i j}=$ $\sigma_{i} d_{i}^{-1}\left(l_{i j}+r_{i j}\right)$ for $i \neq j$ and $q_{i i}=-\sigma_{i} d_{i}^{-1}\left(l_{i}+r_{i}\right)$. The limit here is strong and almost uniform in $t \in(0, \infty)$; for $f \in C_{0}(S)$, the formula holds for $t=0$, as well, and the limit is almost uniform in $t \in[0, \infty)$.

$Q$ is the intensity matrix of the limit Markov chain; the limit semigroup is in general sub-stochastic, since the rows of $Q$ do not necessarily ad up to 0 .

It is worth noting that from the perspective of the theory of semigroups of operators, this theorem is an example of degenerate convergence and by nature it cannot be derived from the Trotter-Kato theorem, see e.g., [3,6,7]. It is also by nature that the limit is almost uniform in $t \in[0, \infty)$ for $f \in C_{0}(S)$ and almost uniform in $t \in(0, \infty)$ for the remaining $f$, see [4]. Since $P \mathrm{e}^{t Q} P f=$ $\mathrm{e}^{t Q} P$, the operators $S(t)=\mathrm{e}^{t Q} P, t \geq 0$ appearing on the right-hand side of (2.6) form a semigroup; however, this semigroup is degenerate in the sense that $\lim _{t \rightarrow 0} S(t) f=f$ merely for $f \in C_{0}(S)$. See e.g., [1-3,6-8,22,32] for examples of such convergence and of such semigroups.

As an immediate corollary of Theorem 2.2, we obtain that any finite-state (possibly not honest) Markov chain is a limit of fast diffusions on a graph in the following sense. Let $Q$ be a (sub-stochastic) $N \times N$ intensity matrix, and let $\mathcal{G}$ be the star-shaped graph with $N$ edges, all vertices but one of degree 1, the special vertex with degree $N$ (see Fig. 3), and all the edges of length 1. We number the edges clockwise, from 1 to $N$, and agree that the special vertex is the left end of all the edges. Finally, we take $\sigma_{i}=1, r_{i}=0$ and $l_{i j}=q_{i j}$. Then, by Theorem 2.2 , the related diffusions on $\mathcal{G}$ with arbitrary choice of $\kappa_{n}$ converge to the Markov chain on the vertices of the related line graph (these vertices may be identified with natural numbers $1, \ldots, N$ ) with intensity matrix $Q$. 


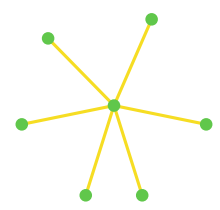

FiguRE 3. Star-shaped graph with 6 edges

\section{Proof of the main result}

The key to the proof is to pass to similar (or: isomorphic) semigroups $[5,12]$. To this end, for $i \in \mathcal{N}$, we define $F_{1, i}, F_{\mathrm{r}, i} \in[C(S)]^{*}$ by

$$
F_{1, i} f=\left[l_{i} f\left(V_{i}\right)-\sum_{j \neq i} l_{i j} f\left(V_{j}\right)\right] ; \quad F_{\mathrm{r}, i} f=-\left[r_{i} f\left(V_{i}\right)-\sum_{j \neq i} r_{i j} f\left(V_{j}\right)\right],
$$

'l' and 'r' standing for 'left' and 'right', respectively. Also, given $\epsilon>0$, we find $\psi_{\mathrm{l}, i}, \psi_{\mathrm{r}, i} \in C\left(E_{i}\right)$ such that $\left\|\psi_{\mathrm{l}, i}\right\|,\left\|\psi_{\mathrm{r}, i}\right\|<\epsilon, \psi_{\mathrm{l}, i}$ and $\psi_{\mathrm{r}, i}$ vanish at both ends of $E_{i}$ while

$$
\psi_{1, i}^{\prime}\left(L_{i}\right)=\psi_{\mathrm{r}, i}^{\prime}\left(R_{i}\right)=1, \quad \psi_{1, i}^{\prime}\left(R_{i}\right)=\psi_{\mathrm{r}, i}^{\prime}\left(L_{i}\right)=0 .
$$

Then, we define $J \in \mathcal{L}(C(S))$ by

$$
J f=\left(\left(F_{1, i} f\right) \psi_{\mathrm{l}, i}+\left(F_{\mathrm{r}, i} f\right) \psi_{\mathrm{r}, i}\right)_{i \in \mathcal{N}} .
$$

Lemma 3.1. For $\kappa \neq 0$, the map $I_{\kappa}=I_{C(S)}-\kappa^{-1} J$ is an isomorphism of $C(S)$ with $I_{\kappa}^{-1}=I_{C(S)}+\kappa^{-1} J$, where $I_{C(S)}$ is the identity operator in $C(S)$.

Proof. Since all $\psi$ 's vanish at the edges' ends

$$
F_{1, i} \circ J=0 \text {, and } F_{\mathrm{r}, i} \circ J=0, \quad i \in \mathcal{N} .
$$

Clearly, $I_{\kappa}$ is linear and bounded. To show that $I_{\kappa}$ is injective, we assume that $I_{\kappa} f=I_{\kappa} g$ for some $f, g \in C(S)$. Applying $F_{1, i}$ to both sides of this relation, by (3.4), we obtain $F_{1, i} f=F_{1, i} g$; similarly, $F_{\mathrm{r}, i} f=F_{\mathrm{r}, i} g$. Therefore, $J f=J g$, and this coupled with $I_{\kappa} f=I_{\kappa} g$ implies $f=g$.

Finally, given $g \in C(S)$, we define $f=g+\kappa^{-1} J g$ to see that, by (3.4), $F_{1, i} f=F_{1, i} g$ and $F_{\mathrm{r}, i} f=F_{\mathrm{r}, i} g$, and so $J f=J g$. Then, $I_{\kappa} f=\left(g+\kappa^{-1} J g\right)-$ $\kappa^{-1} J f=g$, proving that $I_{\kappa}$ is onto and establishing the formula for $I_{\kappa}^{-1}$.

We will also need the following basic information on the one-dimensional Laplacian with Neumann boundary conditions; see e.g., [9].

Lemma 3.2. For real $a<b$, let $G$ in $C[a, b]$ be given by $G f=f^{\prime \prime}$ on the domain composed of twice continuously differentiable functions satisfying $f^{\prime}(a)$ $=f^{\prime}(b)=0$.

(a) $G$ generates a conservative Feller semigroup $\left\{\mathrm{e}^{t G}, t \geq 0\right\}$ in $C[a, b]$.

(b) $G$ is a sectorial operator with angle $\pi / 2$ : for each $\delta \in(0, \pi / 2]$, the sector

$$
\Sigma_{\frac{\pi}{2}+\delta}=\left\{\lambda \in \mathbb{C} \backslash\{0\} ;|\arg \lambda|<\frac{\pi}{2}+\delta\right\}
$$


is contained in the resolvent set $\rho(G)$ of $G$, and there exists $M_{\delta}>0$ such that

$$
\left\|(\lambda-G)^{-1}\right\| \leq \frac{M_{\delta}}{|\lambda|}, \quad \lambda \in \Sigma_{\frac{\pi}{2}+\delta}
$$

(c) $\lim _{n \rightarrow \infty} \lambda\left(\lambda-\kappa_{n} G\right)^{-1} f=\frac{1}{b-a} \int_{a}^{b} f, \lambda>0$, where $\int_{a}^{b} f$ is identified with the constant function on $[a, b]$.

Let $G_{i}$ be the version of $G$ in $C\left(E_{i}\right)$, and let $B$ in $C(S)$ be defined by $B f=\left(\sigma_{i} G_{i} f_{i}\right)_{i \in \mathcal{N}}$ on the Cartesian product of domains of $G_{i}$. Equivalently, $B f=\sigma f^{\prime \prime}$ for all $f \in C^{2}(S)$ satisfying the Neumann boundary conditions $f^{\prime}\left(L_{i}\right)=f^{\prime}\left(R_{i}\right)=0, i \in \mathcal{N}$. Then, $B$ is sectorial because $(3.5)$ is true when $G$ is replaced by $B$, or in fact by any scalar multiple of $B$. Moreover, $B$ generates the strongly continuous, bounded holomorphic, conservative Feller semigroup $\mathrm{e}^{t B}\left(f_{i}\right)_{i \in \mathcal{N}}=\left(\mathrm{e}^{t \sigma_{i} G_{i}} f_{i}\right)_{i \in \mathcal{N}}$ in $C(S)$, and by Lemma $3.2(\mathrm{c})$,

$$
\lim _{n \rightarrow \infty} \lambda\left(\lambda-\kappa_{n} B\right)^{-1} f=P f
$$

where $P$ was defined in Theorem 2.2.

With these preparations, we are ready to prove Proposition 2.1 and Theorem 2.2. Let $B_{n}=I_{\kappa_{n}} A_{n} I_{\kappa_{n}}^{-1}$. For $f \in D\left(A_{n}\right), I_{\kappa_{n}} f$ is twice continuously differentiable, since the $\psi$ 's are, and conditions (2.2) and (2.3) (compare (3.1)) imply $I_{\kappa_{n}} f \in D(B)$. Since the converse implication is also true, $f \in D\left(A_{n}\right)$ iff $I_{\kappa_{n}} f \in D(B)$. This shows that $D\left(B_{n}\right)=D(B)$.

Moreover, for $f \in D(B)$,

$$
A_{n} I_{\kappa_{n}}^{-1} f=\kappa_{n} \sigma f^{\prime \prime}+\sigma(J f)^{\prime \prime}=\kappa_{n} \sigma f^{\prime \prime}+\sigma K f
$$

where $K f$ is given by the right-hand side of (3.3) with $\psi$ 's replaced by their second derivatives. Thus,

$$
B_{n} f=\kappa_{n} B f+C f+D_{n} f
$$

where $C f=-J\left(\sigma f^{\prime \prime}\right)=-J B f$ and $D_{n} f=\sigma K f-\frac{1}{\kappa_{n}} J(\sigma K f)$.

Since $\left\|\psi_{i j}\right\|<\epsilon$, for $\alpha:=\max _{i \in \mathcal{N}}\left\{\left\|F_{1, i}\right\|+\left\|F_{\mathrm{r}, i}\right\|\right\}$, we have $\|J\|<\alpha \epsilon$. Therefore,

$$
\|C f\| \leq \alpha \epsilon\|B f\| \leq \frac{\alpha \epsilon}{\kappa_{n}}\left\|\kappa_{n} B f\right\|, \quad f \in D(B) .
$$

Calculating as in Lemmas 2.5 and 2.6 in [11, pp. 172-173], we obtain for $\lambda \in \Sigma_{\pi / 2+\delta}$,

$$
\begin{aligned}
\left\|C\left(\lambda-\kappa_{n} B\right)^{-1}\right\| & \leq \alpha \epsilon \kappa_{n}^{-1}\left\|\kappa_{n} B\left(\lambda-\kappa_{n} B\right)^{-1}\right\| \\
& =\alpha \epsilon \kappa_{n}^{-1}\left\|\lambda\left(\lambda-\kappa_{n} B\right)^{-1}-I_{C(S)}\right\| \\
& \leq \alpha \epsilon \kappa_{n}^{-1}\left(M_{\delta}+1\right)=: q_{n} \leq \alpha \epsilon \tilde{\kappa}^{-1}\left(M_{\delta}+1\right)=: q<1,
\end{aligned}
$$


provided $\epsilon<\left[\alpha\left(M_{\delta}+1\right)\right]^{-1} \tilde{\kappa}$ where $\tilde{\kappa}=\min _{n \geq 1} \kappa_{n}$. (We used (3.5) with $G$ replaced by $\kappa_{n} B$.) Therefore, $\lambda \in \rho\left(\kappa_{n} B+C\right)$,

$$
\begin{aligned}
\left(\lambda-\kappa_{n} B-C\right)^{-1} & =\left(\lambda-\kappa_{n} B\right)^{-1} \sum_{i=0}^{\infty}\left[C\left(\lambda-\kappa_{n} B\right)^{-1}\right]^{i} \quad \text { and } \\
\left\|\left(\lambda-\kappa_{n} B-C\right)^{-1}\right\| & =\left\|\left(\lambda-\kappa_{n} B\right)^{-1} \sum_{i=0}^{\infty}\left[C\left(\lambda-\kappa_{n} B\right)^{-1}\right]^{i}\right\| \leq \frac{M_{\delta}}{1-q}|\lambda|^{-1} .
\end{aligned}
$$

It follows that the operator $\kappa_{n} B+C$ is sectorial and, being densely defined, generates a holomorphic semigroup. Since $D_{n}$ is bounded, $B_{n}$ generates such a semigroup also and so does $A_{n}$ : we have $\mathrm{e}^{t A_{n}}=I_{\kappa_{n}}^{-1} \mathrm{e}^{t B_{n}} I_{\kappa_{n}}$. In particular, for sufficiently large real $\lambda$, Range $\left(\lambda I_{C(S)}-A_{n}\right)=C(S)$. Since $A_{n}$ can be proved to satisfy the positive maximum principle (comp. [9]), it follows that the semigroup generated by $A_{n}$ is a Feller semigroup [5,13]. Also, this semigroup is conservative iff $1_{S} \in D\left(A_{n}\right)$, that is, iff (2.5) holds. Choosing $\kappa_{n}=1$ for some $n$, we complete the proof of Proposition 2.1.

However, (3.9) shows much more: the semigroups generated by $\kappa_{n} B+C$ are uniformly holomorphic. Therefore, convergence of their resolvents implies convergence of the semigroups [3]. Since $\lim _{n \rightarrow \infty} q_{n}=0$, using (3.7), (3.8) and the Lebesgue dominated convergence theorem, $\lim _{n \rightarrow \infty}\left(\lambda-\kappa_{n} B-C\right)^{-1}=$ $\lim _{n \rightarrow \infty}\left(\lambda-\kappa_{n} B\right)^{-1}$, which by (3.6) equals $\lambda^{-1} P, \lambda>0$. Thus, we have $\lim _{n \rightarrow \infty} \mathrm{e}^{\left(\kappa_{n} B+C\right) t}=P, t>0$. Finally, $D_{n}$ are bounded linear operators converging strongly to $\sigma K$. Hence, using the Phillips perturbation theorem and arguing as in [6, pp. 363-364], we obtain

$$
\lim _{n \rightarrow \infty} \mathrm{e}^{t B_{n}}=\mathrm{e}^{t \sigma P K} P=\mathrm{e}^{t Q} P, t>0
$$

because, calculating:

$$
\begin{aligned}
\sigma P K f & =\left(\sigma_{i} d_{i}^{-1}\left(F_{\mathrm{r}, i} f-F_{1, i} f\right)\right)_{i \in \mathcal{N}} \\
& =\left(\sigma_{i} d_{i}^{-1} \sum_{j \neq i}\left(l_{i j}+r_{i j}\right) f\left(V_{j}\right)-\sigma_{i} d_{i}^{-1}\left(l_{i}+r_{i}\right) f\left(V_{i}\right)\right)_{i \in \mathcal{N}}
\end{aligned}
$$

we see that $\sigma P K$ as restricted to $C_{0}(S)$ equals $Q$. Since $\mathrm{e}^{t A_{n}}=I_{\kappa_{n}}^{-1} \mathrm{e}^{t B_{n}} I_{\kappa_{n}}$ and $\lim _{n \rightarrow \infty} I_{\kappa_{n}}=\lim _{n \rightarrow \infty} I_{\kappa_{n}}^{-1}=I_{C(S)}$, this completes the proof of $(2.6)$.

\section{Acknowledgments}

I am grateful to T. Komorowski, J. Żurawiecki and T. Lipniacki for bibliographical remarks. Moreover, I would like to thank an anonymous referee for his comments on the paper and for relevant references to literature.

Open Access. This article is distributed under the terms of the Creative Commons Attribution License which permits any use, distribution, and reproduction in any medium, provided the original author(s) and the source are credited. 


\section{References}

[1] Banasiak, J., Bobrowski, A.: Interplay between degenerate convergence of semigroups and asymptotic analysis: a study of a singularly perturbed abstract telegraph system. J. Evol. Equ. 9(2), 293-314 (2009)

[2] Banasiak, J., Goswami, A., Shindin, S.: Aggregation in age and space structured population models: an asymptotic analysis approach. J. Evol. Equ. 11, 121154 (2011)

[3] Bobrowski, A.: Degenerate convergence of semigroups. Semigr. Forum 49(3), 303-327 (1994)

[4] Bobrowski, A.: A note on convergence of semigroups. Ann. Polon. Math. 69(2), 107-127 (1998)

[5] Bobrowski, A.: Functional Analysis for Probability and Stochastic Processes. Cambridge University Press, Cambridge (2005)

[6] Bobrowski, A.: Degenerate convergence of semigroups related to a model of stochastic gene expression. Semigr. Forum 73(3), 345-366 (2006)

[7] Bobrowski, A.: On limitations and insufficiency of the Trotter-Kato theorem. Semigr. Forum 75(2), 317-336 (2007)

[8] Bobrowski, A., Bogucki, R.: Semigroups generated by convex combinations of several Feller generators in models of mathematical biology. Studia Mathematica 189, 287-300 (2008)

[9] Bobrowski, A., Morawska, K.: From a PDE model to an ODE model of dynamics of synaptic depression. (2011) (submitted)

[10] Deo, N.: Graph Theory with Applications to Engineering and Computer Science. Prentice-Hall, Inc., Englewood Cliffs (1974)

[11] Engel, K.-J., Nagel, R.: One-parameter Semigroups for Linear Evolution Equations. Springer, New York (2000)

[12] Engel, K.-J., Nagel, R.: A Short Course on Operator Semigroups. Springer, New York (2006)

[13] Ethier, S.N., Kurtz, T.G.: Markov Processes. Characterization and Convergence. Wiley, New York (1986)

[14] Ewens, W.J.: Mathematical Population Genetics. Springer, New York (1979)

[15] Feller, W.: Diffusion processes in genetics. Proceedings of 2nd Symposium on Probability and Statistics, Berkeley (1950)

[16] Feller, W.: The parabolic differential equations and the associated semi-groups of transformations. Ann. Math. 55, 468-519 (1952)

[17] Feller, W.: Diffusion processes in one dimension. Trans. Am. Math. Soc. 77(1), 1-31 (1954)

[18] Feller, W.: Two singular diffusion problems. Ann. Math. 54, 173-182 (1954)

[19] Fijavž, M.K., Mugnolo, D., Sikolya, E.: Variational and semigroup methods for waves and diffusion in networks. Appl. Math. Optim. 55, 219-240 (2007)

[20] Freidlin, M.I., Sheu, S.-J.: Diffusion processes on graphs: stochastic differential equations, large deviation principle. Probab. Theory Relat. Fields 116, $181-220(2000)$

[21] Freidlin, M.I., Wentzel, A.D.: Random Perturbations of Dynamical Systems, 2nd edn. Springer, Berlin (1998) 
[22] Freidlin, M.I., Wentzel, A.D.: Diffusion processes on graphs and the averaging principle. Ann. Prob. 21, 2215-2245 (1993)

[23] Freidlin, M.I., Wentzel, A.D.: Random Perturbations of Hamiltonian Systems. AMS Mem. Am. Math. Soc. 109(523) (1994)

[24] Ito, K., Mc Kean, H.P. Jr.: Diffusion Processes and their Sample Paths. Springer, Berlin (1996) Reprint of the 1974 Edition. Classics in Mathematics Series

[25] Karatzas, I., Shreve, S.E.: Brownian Motion and Stochastic Calculus. Springer, New York (1991)

[26] Kaźmierczak, B., Lipniacki, T.: Regulation of kinase activity by diffusion and feedback. J. Theor. Biol. 259, 291-296 (2009)

[27] Kostrykin, V., Potthoff, J., Shrader, R.: Contraction semigroups on metric graphs. In: Exner, P., Keating, J., Kuchment, P., Sunada, T., Teplyaev, A. (eds.). Analysis on Graphs and its Applications, vol. 77 of Proceedings of Symposium on Pure Mathematics, pp. 423-458. American Mathematical Society, Providence, R.I. (2008)

[28] Kostrykin, V., Potthoff, J., Shrader, R.: Brownian motions on metric graphs. ArXiv:1102.4937v1 (2011)

[29] Mandl, P.: Analytical Treatment of One-Dimensional Markov Processes. Springer, New York (1968)

[30] Mugnolo, D.: Gaussian estimates for a heat equation on a network. Netw. Het. Media 2, 55-79 (2007)

[31] Mugnolo, D.: Semigroup methods for evolution equations on networks, pp. 1-75. (2007) (preprint)

[32] Nittka, R.: Approximation of the semigroup generated by the Robin Laplacian in terms of the Gaussian semigroup. J. Funct. Anal. 257(5), 1429-1444 (2009)

[33] Revuz, D., Yor, M.: Continuous Martingales and Brownian Motion, 3rd edn. Springer, Berlin (1999)

[34] Taylor, H.M., Karlin, S.: Second Course in Stochastic Processes. Academic Press, London (1981)

Adam Bobrowski

Department of Mathematics

Faculty of Electrical Engineering and Computer Science

Lublin University of Technology

Nadbystrzycka 38A

20-618 Lublin

Poland

e-mail: a.bobrowski@pollub.pl

Communicated by Jan Derezinski

Received: October 24, 2011.

Accepted: December 16, 2011. 